\title{
Cross-section of Asbestos Prepared for TEM/STEM with Ion Slicer
}

\author{
I. Ohnishi, ${ }^{*}$ H. Nishioka, ${ }^{*}$ E. Okunishi, ${ }^{*}$ H. Takahashi, ${ }^{*}$ and Y. Kondo*
}

*JEOL Ltd., 3-1-2 Musashino, Akishima, Tokyo, 196-8558, JAPAN

Ion Slicer [1]: an ion milling tool using a broad argon ion beam, is a thin film preparation instrument for TEM. This produces high quality thin films with little damage and artifact without any special skill. Thin films of the cross-sections of two types of asbestos fibers were prepared with Ion Slicer in this study.

Samples were chrysotile: $\mathrm{Mg}_{3} \mathrm{Si}_{2} \mathrm{O}_{5}(\mathrm{OH})_{4}$ and amosite: $(\mathrm{Mg}, \mathrm{Fe})_{7} \mathrm{Si}_{8} \mathrm{O}_{22}(\mathrm{OH})_{2}$. The former is representative fibrous asbestos, mineralogically belonging to a serpentine group, whereas the latter is acicular one, belonging to an amphibole group. TEM thin films of these samples were made as follows. Asbestos fibers were aligned in the same direction parallel to their longitudinal direction, embedded in epoxy resin and mounted on a silicon wafer. Plates with about 500 micrometers in thickness were cut out from the wafer with the asbestos perpendicular to the longitudinal direction of fibers by a diamond cutter. These plates were thinned down to about 100 micrometers by mechanical polishing to prepare thin plates. From these thin plates were prepared thin films for TEM with Ion Slicer. They were observed with TEMs, JEM-2100 and JEM-2100F.

Fig. 1 shows a high-resolution TEM image of the cross-sections of the chrysotile fibers. Each fiber is a hollow cylinder with its thickness part consisting of either concentric or spiral layers. This morphology is characteristic to serpentine asbestos and the same as the one first observed in TEM by Yada [2] using the thin film prepared with an ultramicrotome. Fig. 2 shows high-resolution STEM images of the cross-section of the amosite. In both BF (bright field) and HAADF (high angle annular dark field) images is observed the I-beam structure that has been discovered by X-ray crystallography [3]. Though these structures were easily destroyed by electron irradiation during observation in TEMs, damage due to the thin film preparation with the argon ion beam was indiscernible in both thin films. Besides high quality high-resolution images of nanometer size areas, a large cross-sectional area with a size of a few square micrometers could also be observed. The present study confirmed again a very high performance of Ion Slicer as a thin film preparation instrument even for fine and brittle fibrous materials such as asbestos.

References

[1] A. Yasuhara, JEOL news 40 (2005) 46.

[2] K. Yada, Acta Cryst. 23 (1967) 704.

[3] A. Putnis, An Introduction to Mineral Sciences, Cambridge University Press, Cambridge, 1992. 


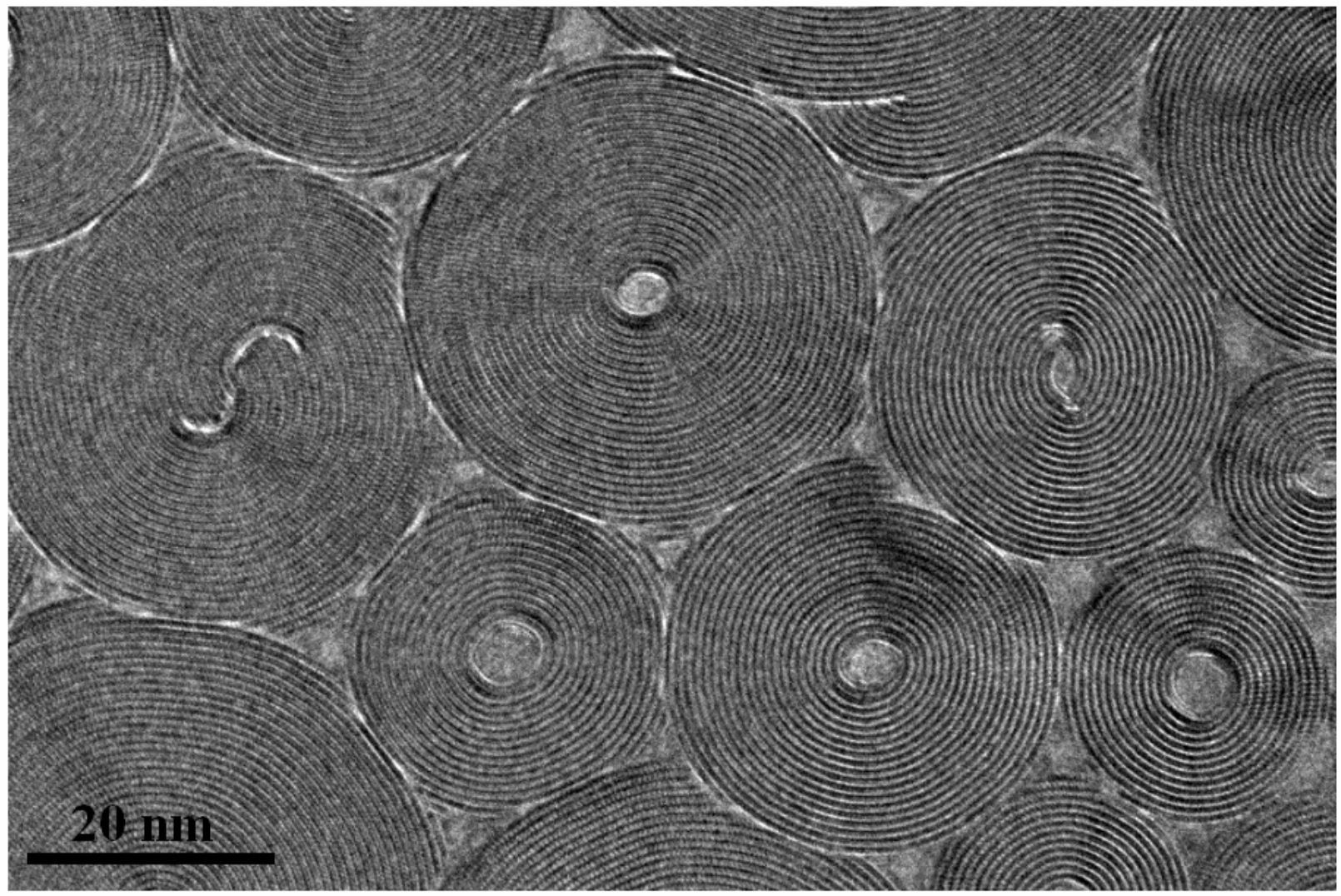

Fig. 1. A high-resolution TEM image of the cross-section of chrysotile asbestos.
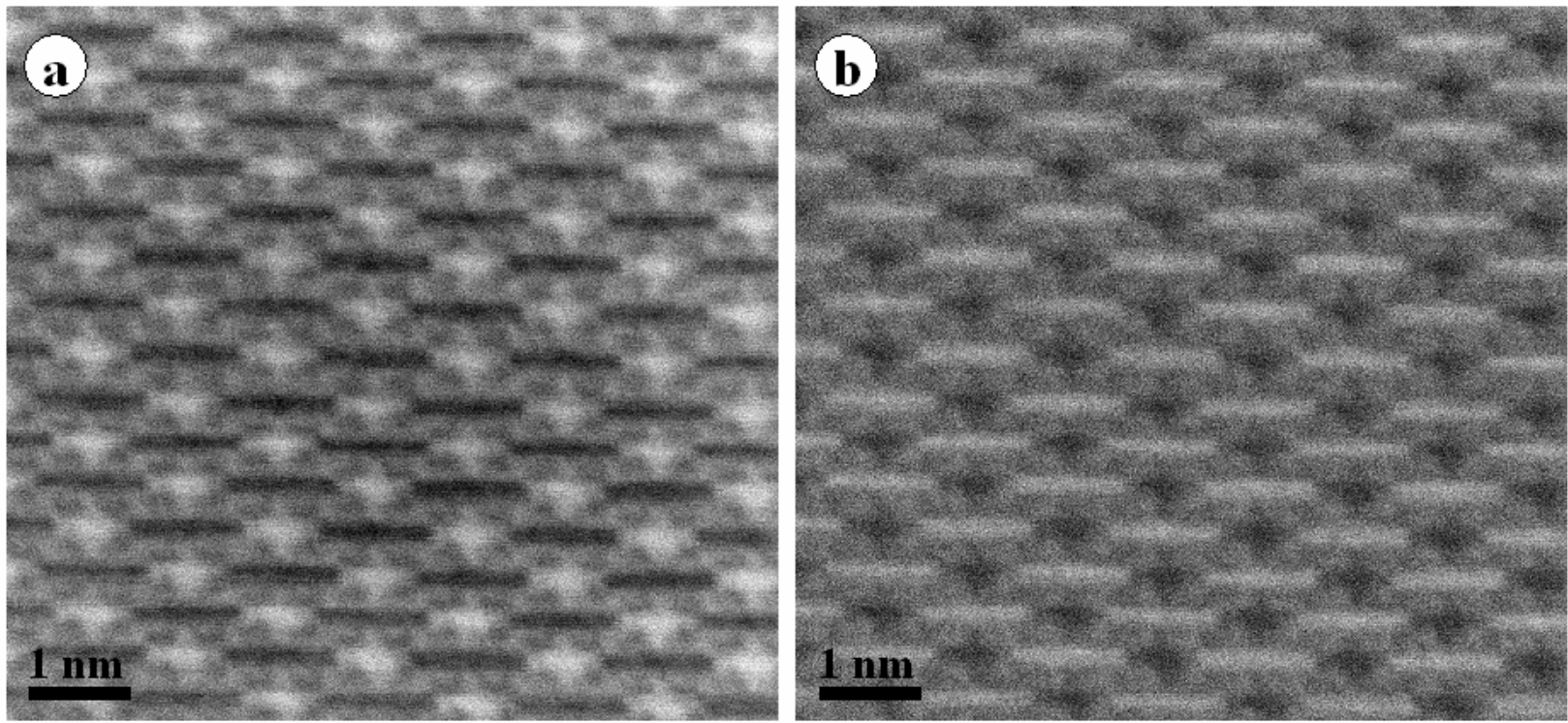

Fig. 2. High-resolution STEM images of the cross-section of amosite asbestos, (a) BF image, (b) HAADF image. 